\title{
Cost-effectiveness analysis of cabazitaxel for metastatic castration resistant prostate cancer after docetaxel and androgen- signaling-targeted inhibitor resistance
}

Peng-Fei Zhang ${ }^{1,2}$, Dan Xie ${ }^{3,4}$ and Qiu Li ${ }^{1,2^{*}}$

\begin{abstract}
Background: The aim of our study was to evaluate the cost-effectiveness of cabazitaxel versus abiraterone or enzalutamide in patients with metastatic castration-resistant prostate cancer (mCRPC) previously treated with docetaxel who had progression within 12 months while receiving an alternative inhibitor (abiraterone or enzalutamide) from a US payer's perspective.
\end{abstract}

Methods: To conduct the cost-effectiveness analysis, a Markov decision model was established. Three health states (progression-free survival (PFS), progressive disease (PD) and death) were included, and the incremental costeffectiveness ratio (ICER) was regarded as the primary endpoint. The willingness-to-pay (WTP) threshold was set at $\$ 100,000.00$ /quality-adjusted life year (QALY), and discounted rates were set at 3\% annually. Efficacy data were derived from the CARD trial and Weibull distribution curves were modeled to fit the survival curves. The robustness of the analysis was tested with a series of one-way sensitivity analyses and probabilistic sensitivity analyses.

Results: Overall, the incremental effectiveness and cost of cabazitaxel versus androgen-signaling-targeted inhibitors (ASTIs) were 0.16 QALYs and \$49,487.03, respectively, which yielded an ICER of $\$ 309,293.94 /$ QALY. Our model was mostly sensitive to the duration of PFS in the cabazitaxel group, cost of cabazitaxel and utility of the PFS state. At a WTP threshold of $\$ 100,000.00 /$ QALY, cabazitaxel was the dominant strategy in $0 \%$ of the simulations.

Conclusions: Cabazitaxel is unlikely to be a cost-effective treatment option compared with ASTIs in patients with mCRPC previously treated with docetaxel who had progression within 12 months while receiving ASTIs.

Keywords: Cabazitaxel, Abiraterone, Enzalutamide, Docetaxel, Cost-effectiveness, Prostate cancer

\footnotetext{
* Correspondence: keythera@126.com

'Department of Medical Oncology, Cancer Center, West China Hospital, Sichuan University, Chengdu, China

${ }^{2}$ West China Biomedical Big Data Center, West China Hospital/West China School of Medicine, Sichuan University, Chengdu, China

Full list of author information is available at the end of the article
}

(c) The Author(s). 2021 Open Access This article is licensed under a Creative Commons Attribution 4.0 International License, which permits use, sharing, adaptation, distribution and reproduction in any medium or format, as long as you give appropriate credit to the original author(s) and the source, provide a link to the Creative Commons licence, and indicate if changes were made. The images or other third party material in this article are included in the article's Creative Commons licence, unless indicated otherwise in a credit line to the material. If material is not included in the article's Creative Commons licence and your intended use is not permitted by statutory regulation or exceeds the permitted use, you will need to obtain permission directly from the copyright holder. To view a copy of this licence, visit http://creativecommons.org/licenses/by/4.0/. The Creative Commons Public Domain Dedication waiver (http://creativecommons.org/publicdomain/zero/1.0/) applies to the data made available in this article, unless otherwise stated in a credit line to the data. 


\section{Background}

Prostate cancer represents the second most commonly diagnosed cancer and the fifth leading cause of cancerrelated deaths in men worldwide [1]. Previously, the standard first-line treatment was androgen- deprivation therapy (ADT), which could be achieved by bilateral orchiectomy or luteinizing hormone-releasing hormone (LHRH) agonists/ antagonists [2]. However, most patients with advanced prostate cancer will become refractory to ADT, which denotes metastatic castration-resistant prostate cancer (mCRPC) [3]. Several novel drugs, such as docetaxel, androgen-signalingtargeted-inhibitors (ASTIs) and sipuleucel-T, have been demonstrated to prolong survival in patients with mCRPC [4-8]. Recently, ASTIs, such as abiraterone and enzalutamide, have also been demonstrated to be effective in the treatment of metastatic hormone-sensitive prostate cancer in combination with ADT [9-12]. Based on these studies, ASTIs (abiraterone or enzalutamide) and docetaxel are frequently used in patients with prostate cancer in earlier stages, and most of the patients are likely to receive both ASTIs and docetaxel, in either order.

Despite the effectiveness of these regimens, most patients will still become refractory. Once this occurs, a switch to a subsequent ASTI or the use of cabazitaxel has been the standard medical practice. Recently, the efficacy of poly (ADP-ribose) polymerase (PARP) inhibitors, such as olaparib and rucaparib, has also been investigated in patients with $\mathrm{mCRPC}$ that harbored alterations in the DNA-damage-repair genes [13, 14]. These studies showed PARP inhibitors could achieve longer survival than a second-generation androgen-deprivation therapy (abiraterone or enzalutamide) in these patients. However, although all these regimens have showed efficacy in the treatment of post-docetaxel mCRPC patients, evidence for the treatment sequence of these drugs is lacking. Based on previous studies, patients may become resistant to abiraterone or enzalutamide after disease progression on previous ASTI treatment [15-17]. Moreover, some evidence also suggests partial cross-resistance between ASTIs and docetaxel [18]. Thus, it would be important to investigate which treatment regimen is superior in patients previously treated with docetaxel and ASTIs. Recently, the results of the CARD trial, which evaluated the efficacy and safety of cabazitaxel versus abiraterone or enzalutamide in patients with mCRPC previously treated with docetaxel who had progression within 12 months while receiving ASTIs, were published [19]. Cabazitaxel significantly prolonged the median imaging-based progression-free survival (PFS) (8.0 months versus 3.7 months) and median overall survival (OS) (13.6 months versus 11.0 months) compared with ASTIs, indicating that cabazitaxel is a more favorable treatment option for patients with mCRPC previously treated with docetaxel and ASTIs.
Despite the benefit achieved by cabazitaxel, the high cost of the treatment may significantly increase healthcare expenditures. Given the heavy healthcare burden worldwide currently, it is crucial to determine which regimen is with better efficiency and pharmacoeconomic profile $[20,21]$. The aim of the study was to evaluate the cost-effectiveness of cabazitaxel versus ASTIs (abiraterone or enzalutamide) in patients with mCRPC previously treated with docetaxel who had progression within 12 months while receiving ASTIs from a US payer's perspective.

\section{Methods \\ Model structure}

A Markov decision model was established to evaluate the cost-effectiveness of cabazitaxel versus ASTIs in patients with mCRPC previously treated with docetaxel who had progression within 12 months while receiving an alternative inhibitor (abiraterone or enzalutamide). PFS, progressive disease (PD) and death were defined as the three mutually exclusive health states in the model. All participants were assumed to enter the model in the PFS state, at the end of each cycle, the patients could stay in the starting health state or transition to the PD state or death. Once in the PD state, patients could remain in that state or transition to death at the end of each cycle [22]. Health utilities for the PFS state and PD state were derived from previously published literature [22]. Adverse events (AEs) in the model were chosen if they occurred with high frequency $(>5 \%)$, were expensive to treat or substantively affected quality of life (Table 1). Regardless of the influence of AEs, the health utility values in the model were assumed to be invariable. Quality-adjusted life-years (QALYs) and cost were measured, and the incremental cost-effectiveness ratio (ICER) of cabazitaxel versus ASTIs was regarded as the primary endpoint in the study. The willingness-to-pay (WTP) threshold in the analysis was set at $\$ 100,000.00$ / QALY [23, 24]. Effectiveness and cost outcomes were discounted at a 3\% annual rate in the model [23, 25]. The time horizon of the model was defined as 10 years, and the Markov cycle length in the model was 3 weeks, which is consistent with the length of the treatment periods. This study was approved by the Ethics Committee of our hospital. The model was developed and tested using the R statistical environment (version 3.6.1; R Development Core Team, Vienna, Austria) and TreeAge software (TreeAge, Williamstown, MA, USA, 2011).

\section{Patients and treatments}

A cohort population reflecting the participants of the CARD trial was modeled in the study [19]. Patients who were confirmed as metastatic prostate cancer histologically and had previously been treated with three or more cycles of docetaxel, and had previously had disease progression 
Table 1 Key clinical data in the model

\begin{tabular}{|c|c|c|c|}
\hline Parameters & Cabazitaxel & ASTI & Reference \\
\hline \multicolumn{4}{|l|}{ Weibull parameters } \\
\hline Scale $(\lambda)$ for PFS & 0.034827 & 0.069115 & [19] \\
\hline Shape $(\gamma)$ for PFS & 1.486146 & 1.468545 & [19] \\
\hline Scale $(\lambda)$ for OS & 0.007118 & 0.007698 & [19] \\
\hline Shape $(\gamma)$ for OS & 1.676228 & 1.867383 & [19] \\
\hline \multicolumn{4}{|l|}{ Survival } \\
\hline Median OS (range), month & $13.6(11.5-17.5)$ & $11.0(9.2-12.9)$ & [19] \\
\hline Median imaging PFS (range), month & $8.0(5.7-9.2)$ & $3.7(2.8-5.1)$ & [19] \\
\hline Median PFS (range), month & $4.4(3.6-5.4)$ & $2.7(2.4-2.8)$ & [19] \\
\hline \multicolumn{4}{|l|}{ Treatment } \\
\hline Median treatment duration (range), month & $22(3-88)$ & $12.5(2-141)$ & [19] \\
\hline Median treatment cycles & $7(1-29)$ & $4(1-45)$ & [19] \\
\hline \multicolumn{4}{|l|}{ Probability of AEs (grade 3/4) } \\
\hline Musculoskeletal pain or discomfort & $0.016(0.013-0.019)$ & $0.056(0.045-0.067)$ & [19] \\
\hline Renal disorder & $0.032(0.026-0.038)$ & $0.081(0.065-0.097)$ & [19] \\
\hline Anemia & $0.080(0.064-0.096)$ & $0.048(0.038-0.058)$ & [19] \\
\hline Leukopenia & $0.320(0.256-0.384)$ & $0.016(0.013-0.019)$ & [19] \\
\hline Neutropenia & $0.447(0.358-0.536)$ & $0.032(0.026-0.038)$ & [19] \\
\hline \multicolumn{4}{|l|}{ Utility } \\
\hline PFS & $0.617(0.494-0.740)$ & $0.617(0.494-0.740)$ & {$[22]$} \\
\hline PD & $0.370(0.296-0.444)$ & $0.370(0.296-0.444)$ & {$[22]$} \\
\hline
\end{tabular}

ASTI androgen-signaling-targeted inhibitor, PFS progression-free survival, OS overall survival, AEs adverse events

during 12 months of treatment with an androgensignaling-targeted inhibitor, were included. Cabazitaxel was administered intravenously at a dose of $25 \mathrm{mg}$ per square meter of body surface area (BSA) every 3 weeks. Meanwhile, these patients also received $10 \mathrm{mg}$ oral prednisone daily. Based on the results of the CARD trial, of the 124 patients who received an ASTI, 58 received abiraterone and 66 received enzalutamide. Abiraterone was given at $1000 \mathrm{mg}$ orally once daily, and oral prednisone was given at $5 \mathrm{mg}$ twice daily, while enzalutamide was administered orally at a dose of $160 \mathrm{mg}$ once daily every 3 weeks. Abiraterone was given to enzalutamide-resistant patients, and enzalutamide was given to patients who failed previous abiraterone treatment.

\section{Efficacy inputs}

Efficacy data in the model were derived from the CARD trial, and the information was used to estimate the transition probabilities between health states. Survival data points were extracted from the survival curves using a plot digitizer software (DigitizeIt, version 2.0, Braunschweig, Germany, www.digitizeit.de). Weibull distribution curves were modeled to fit the survival curves in the CARD trial. The fitting Weibull parameters (scale $(\lambda)$ and shape $(\gamma)$ ) are presented in Table 1, and the calibration curves are shown in Fig. 1. The probabilities of progression and death were estimated from the curves as described by a previous study [26].

\section{Cost and resource data}

The cost in the model was estimated from the perspective of US payers, and the costs estimated in the trial are presented in Table 2. The costs of drugs, drug administration, follow-up (reflecting the frequency of drug administration), tests, AE-related treatments and best supportive care (BSC) were included in the model. The unit prices of drugs were obtained from the average whole prices from Red Book Online ${ }^{\oplus}$, an online resource that lists drug product pricing of medications in the US. To calculate the dose of cabazitaxel, a patient with a height of $176 \mathrm{~cm}$ and weight of $88.9 \mathrm{~kg}$ (BSA of $2.1 \mathrm{~m}^{2}$ ) was assumed [30]. The unit cost of tests, drug administration, follow-up, tests, AE-related treatments and BSC were retrieved from the Centers for Medicare \& Medicaid (CMS) clinical laboratory fee schedule files and previously published literature [27-30]. The median number of treatment cycles was 7 in the cabazitaxel group and 4 in the ASTI group. AE-related cost was calculated by multiplying the incidence of AEs by the cost of managing these AEs per event. 
A

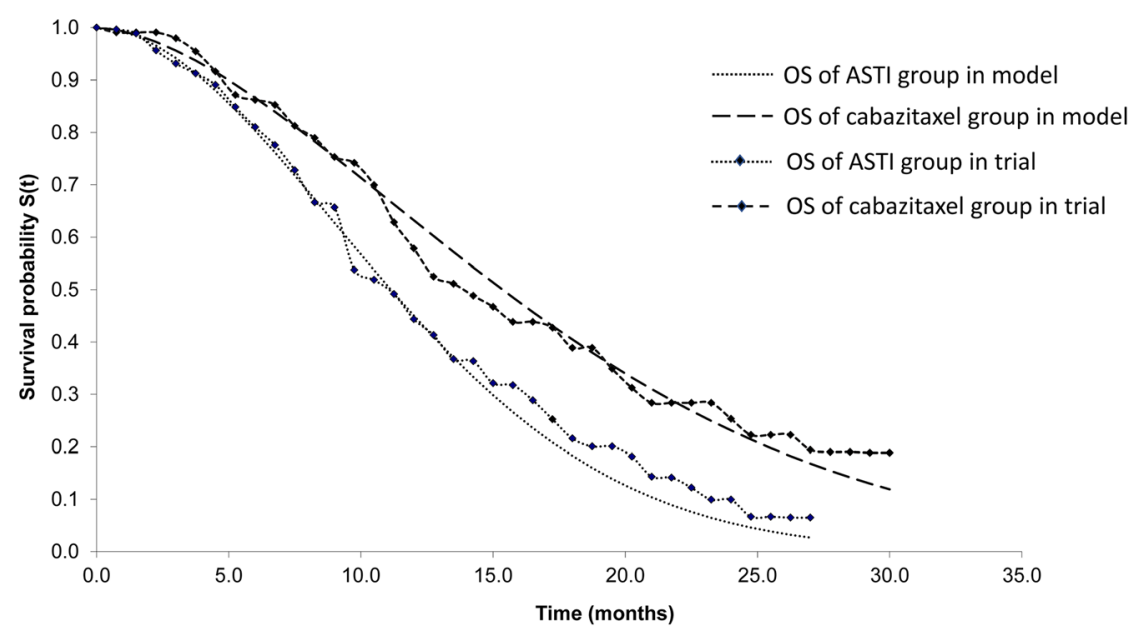

B

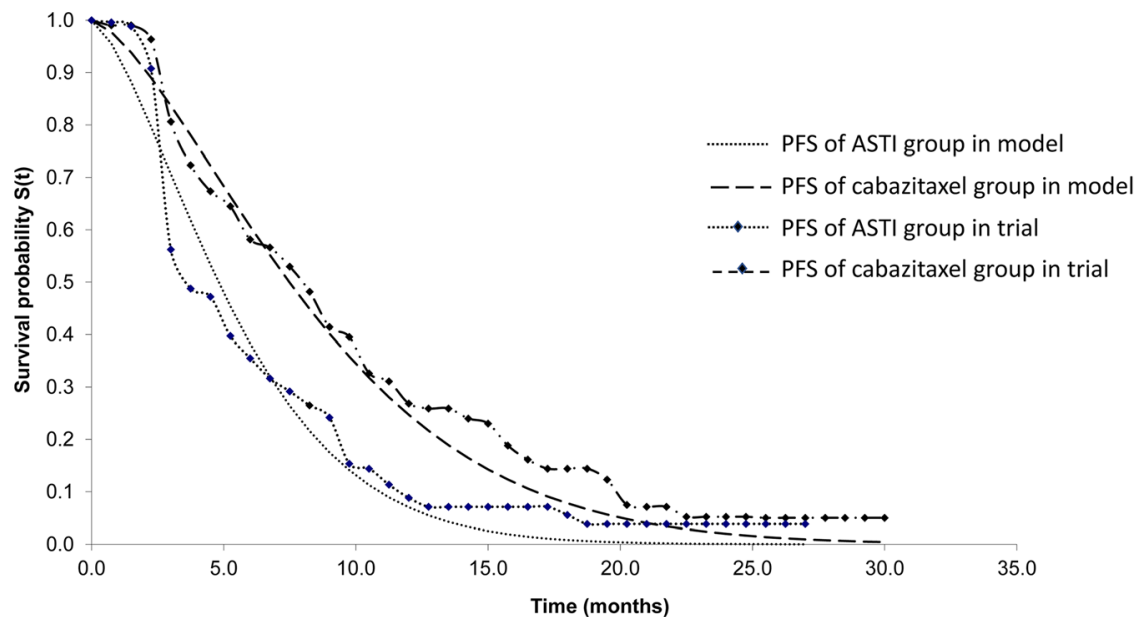

Fig. 1 Estimated Weibull survival curves for cabazitaxel group (a) and ASTI group (b). PFS, progression-free survival; OS, overall survival; ASTI, androgen-signaling-targeted inhibitor

\section{Sensitivity analysis}

The robustness of the analysis was tested with a series of one-way sensitivity analyses on several parameters. In the one-way sensitivity analyses, parameters ranged between -20 and $+20 \%$, and the results of the one-way sensitivity analyses are presented as tornado diagrams. Moreover, probabilistic sensitivity analyses were also performed based on Monte Carlo simulations with 1000 iterations. Gamma distributions were used for cost inputs and beta distributions were used for the probabilities of AEs and utility values. The results of the analyses are shown as cost-effectiveness acceptability curves.

\section{Results}

\section{Base case analysis}

Over a 10-year time lifetime horizon, cabazitaxel produced an effectiveness of 0.57 QALYs per patient at a total cost of $\$ 105,169.70$, while ASTIs achieved an effectiveness of
0.41 QALYs per patient at a cost of $\$ 55,682.67$. Overall, the incremental effectiveness and cost of cabazitaxel versus ASTIs were 0.16 QALYs and $\$ 49,487.03$ per patient, respectively, which yielded an ICER of \$309,293.94/QALY gained with cabazitaxel versus ASTIs (Table 3).

\section{Sensitivity analyses}

The results of the one-way sensitivity analyses are presented in Fig. 2. The top 3 parameters that mostly impacted the ICER were duration of PFS in the cabazitaxel group, cost of cabazitaxel and utility of the PFS state. The ICER was also sensitive to the duration of PFS in the ASTI group and cost of ASTIs. Other parameters, such as cost of drug administration and cost of follow-up, had little impact on the results of our analysis. In the probabilistic sensitivity analyses, the proportion of cabazitaxel as the more cost-effective strategy compared with ASTIs was $0 \%$ when the WTP threshold was set at \$100,000.00/QALY (Fig. 3). 
Table 2 Cost parameters input in the model

\begin{tabular}{lll}
\hline Parameters & Value (range) & Resource \\
\hline Cabazitaxel $(60 \mathrm{mg})$ & $13,170.31(10,536.248-15,804.384)$ & RED BOOK \\
Abiraterone $(250 \mathrm{mg})$ & $95.26(76.208-114.312)$ & RED BOOK \\
Enzalutamide $(40 \mathrm{mg})$ & $115.486(92.389-138.583)$ & RED BOOK \\
Prednisone $(10 \mathrm{mg})$ & $0.53(0.424-0.636)$ & RED BOOK \\
Prednisone $(5 \mathrm{mg})$ & $0.39(0.312-0.468)$ & RED BOOK \\
Administration per unit CPT:96365 & $74.16(59.328-88.992)$ & CMS \\
Musculoskeletal pain or discomfort per event & $364.8(291.84-437.76)$ & [26], 2015 \\
Renal disorder per event & $5966.67(4773.336-7160.004)$ & [27], 2015 \\
Anemia per event & $1881(1826-1910)$ & [27], 2015 \\
Leukopenia per event & $3066(2758-3384)$ & [27], 2015 \\
Neutropenia per event & $3066(2758-3384)$ & [27], 2015 \\
Laboratory tests per event & $76(68-84)$ & [27], 2015 \\
Chest/Abdomen/Pelvis CT per event & $828(598-1083)$ & [27], 2015 \\
PSA per event & $25(20-30)$ & [28], 2015 \\
Bone scanning per event & $253.46(202.768-304.152)$ & [28], 2015 \\
Cost of supportive care per cycle & $1213(987-1438)$ & [29], 2018 \\
Routine follow-up of patients per unit & $422(348.1-495.8)$ & [29], 2018 \\
\hline
\end{tabular}

CT Computed Tomography, PSA prostate specific antigen

\section{Discussion}

Prostate cancer remains one of the most common malignancies in men worldwide. In recent years, ADT combined with docetaxel, abiraterone, or enzalutamide, have been demonstrated to be effective treatment options for patients with metastatic hormone-sensitive prostate cancer. However, for patients with mCRPC previously treated with docetaxel who had progression within 12 months while receiving an alternative inhibitor (abiraterone or enzalutamide), evidence for the treatment sequence of drugs is lacking. In the CARD trial, cabazitaxel was demonstrated to significantly prolong survivalcompared

Table 3 Base case results of the model

\begin{tabular}{lll}
\hline Model outcomes & Cabazitaxel & ASTI \\
\hline Cost (\$) & & \\
Costs in PFS & $94,672.44$ & $43,831.50$ \\
Costs in PD & $10,497.26$ & $11,851.17$ \\
Total costs & $105,169.70$ & $55,682.67$ \\
Incremental costs & $49,487.03$ & - \\
Effectiveness (QALYs) & & \\
QALYs in PFS & 0.40 & 0.19 \\
QALYs in PD & 0.17 & 0.22 \\
Total effectiveness & 0.57 & 0.41 \\
Incremental effectiveness & 0.16 & - \\
ICER (\$/QALY) & $309,293.94$ & \\
\hline
\end{tabular}

ASTI androgen-signaling-targeted inhibitor, PFS progression-free survival, OS overall survival, $Q A L Y$ s quality-adjusted life years, IECR incremental cost-effectiveness ratio with the ASTIs, which suggested cabazitaxel as the optimal treatment option for patients with MCRPC who had been previously treated with docetaxel and ASTIs.

In this study, however, we evaluated the benefit of cabazitaxel versus ASTIs in patients with mCRPC previously treated with docetaxel and alternative inhibitors from a pharmacoeconomic perspective. Overall, cabazitaxel achieved more effectiveness than ASTIs (0.57 QALYs versus 0.41 QALYs); however, the cost in the cabazitaxel group was much higher than that in the ASTI group $(\$ 105,169.70$ versus $\$ 55,682.67)$. The ICER of cabazitaxel versus ASTIs was $\$ 309,293.94 /$ QALY. Based on the WTP threshold of $\$ 100,000.00 /$ QALY, cabazitaxel might not be a costeffective treatment option compared with ASTIs.

In the sensitivity analyses, the parameters having the greatest influence on the ICER were the duration of PFS in the cabazitaxel group, cost of cabazitaxel, utility of the PFS state, the duration of PFS in the ASTI group and cost of ASTIs. Thus, selecting the subgroup of patients with the best survival benefit of cabazitaxel is essential to improve the pharmacoeconomic profile of cabazitaxel versus ASTIs. On the other hand, cost of cabazitaxel and cost of ASTIs were two other key parameters, which was likely because of their high prices. In addition, the median duration of treatment was longer in patients receiving cabazitaxel than in those receiving ASTIs ( 22.0 weeks vs. 12.5 weeks), and the median number of treatment cycles received was also higher in patients receiving cabazitaxel than in those receiving ASTIs (7 vs. 4 ), which reflected the higher disease progression rates 


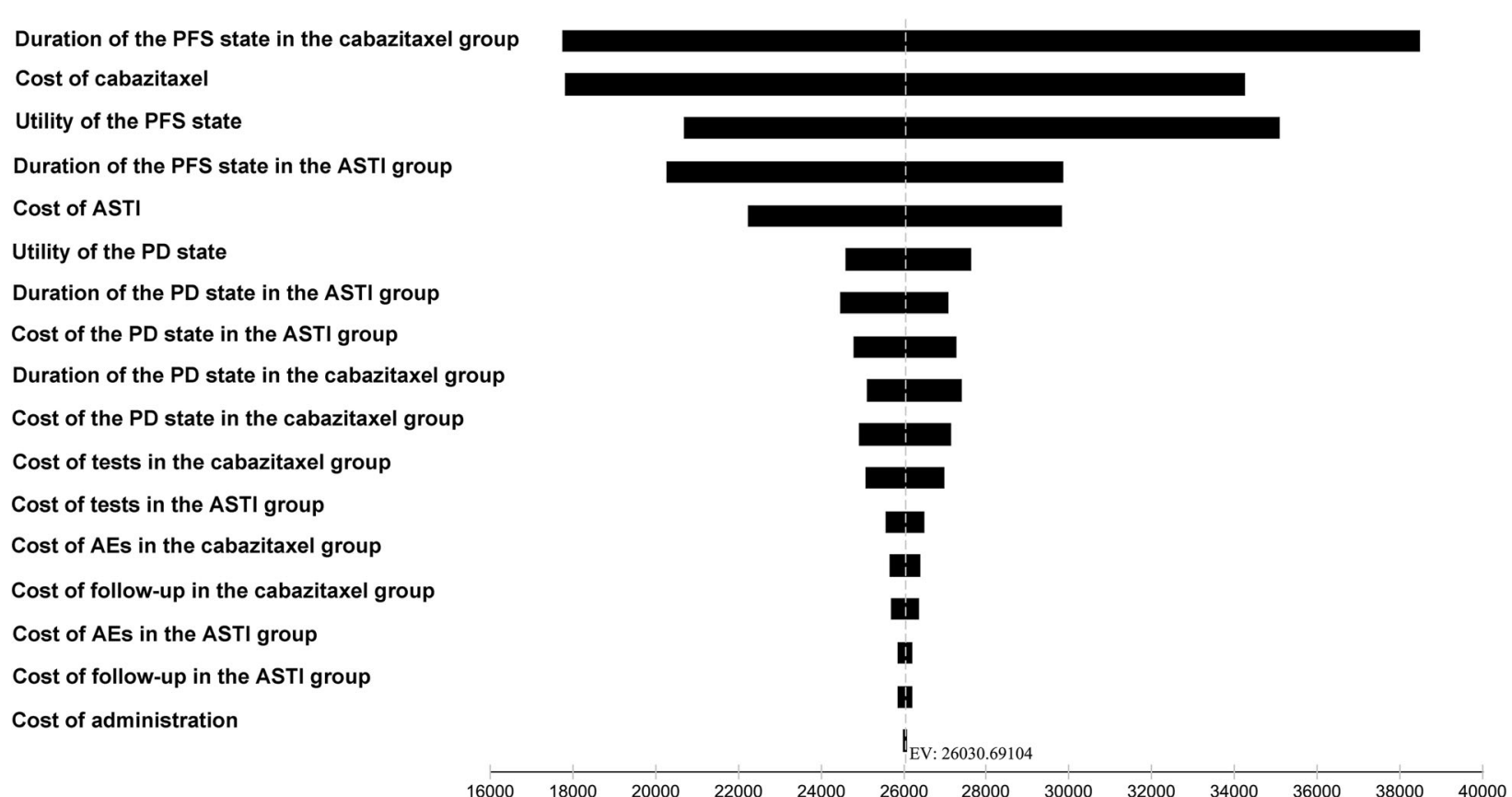

Fig. 2 Tornado diagram for one-way sensitivity analyses. PFS, progression-free survival; PD, progressive disease; AEs, adverse events; ICER, incremental cost-effectiveness ratio; ASTI, androgen-signaling-targeted inhibitor

(in 43.7 and $71.0 \%$ of the patients, respectively) and poorer outcomes in the ASTI group.

To the best of our knowledge, this is the first economic analysis to evaluate the cost-effectiveness of cabazitaxel versus ASTIs for patients with MCRPC who had been previously treated with docetaxel and alternative androgen-signaling-targeted agents using a Markov model. In a recent study, investigators compared abiraterone plus prednisone (ABI+PRD), cabazitaxel plus prednisone $(\mathrm{CAB}+\mathrm{PRD})$ and enzalutamide (ENZ) for visceral metastatic CRPC post-docetaxel therapy resistance development [22]. The LYs and QALYs were 1.20 and 0.58 , respectively, for $A B I+P R D, 1.48$ and 0.56 for $\mathrm{CAB}+\mathrm{PRD}$, and 1.58 and 0.79 for ENZ. The total treatment cost was: $\$ 115,433$ for $A B I+P R D, \$ 85,337$ for $\mathrm{CAB}+\mathrm{PRD}$ and $\$ 109,213$ for ENZ. CAB+PRD and ENZ were superior to $A B I+P R D$ due to the higher LYs gained. This analysis found that ENZ provided greater LYs and QALYs than both ABI+PRD and CAB+PRD at a lower cost than $\mathrm{ABI}+\mathrm{PRD}$, but at a higher cost than $\mathrm{CAB}+$

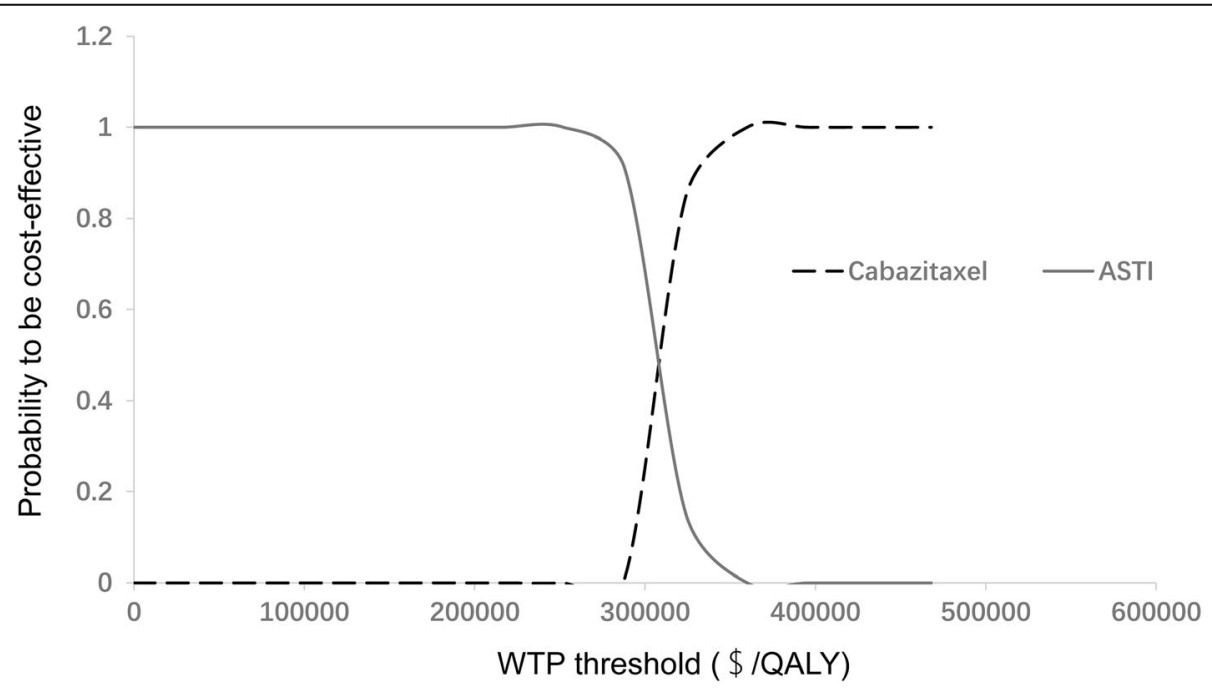

Fig. 3 The cost-effectiveness acceptability curves for probabilistic sensitivity analyses. QALY, quality-adjusted life year; WTP, willingness-to-pay 
PRD. For patients with visceral mCRPC after docetaxel therapy resistance, ENZ was cost-effective $92 \%$ of the time with a WTP threshold of $\$ 100,000 /$ QALY. The cost and effectiveness of the cabazitaxel group in the above study were similar to those in our study; however, the cost and effectiveness in the abiraterone and enzalutamide groups were much higher than those in our analysis. This could be explained by the fact that the above study was conducted based on the results of three studies, the TROPIC trial that compared CAB+PRD to mitoxantrone plus prednisone [5], the COU-AA-301 trial that compared $\mathrm{ABI}+\mathrm{PRD}$ to placebo plus prednisone [7], and the AFFIRM trial that compared ENZ to placebo plus prednisone [8]. The median durations of treatment were 8 months and 8.3 months in the groups that received abiraterone acetate plus prednisone and enzalutamide, respectively, which were higher than the median number of treatment cycles in the CARD trial. However, the number of treatment cycles of patients in the cabazitaxel group in the TROPIC trial was similar to the number of cycles in the CARD trial.

In addition to cabazitaxel, other novel drugs, were also investigated in the setting of metastatic castrationresistant prostate cancer who had disease progression while receiving a new hormonal agent (e.g., enzalutamide or abiraterone). $\mathrm{mCRPC}$ is a heterogeneous disease and a series of genomic aberrations have been identified, which included deleterious aberrations in genes involved in repairing DNA damage [31,32]. Germline or somatic mutations in DNA damage repair (DDR) genes, such as BRCA2, CHEK2, ATM, RAD51D, BRCA1, and PALB2, have been described in around $20-25 \%$ of advanced prostate cancer patients, which involved in aggressive disease and poor outcomes in these patients [31]. However, they also might be therapeutic targets. Recently, the effect of a series of PARP inhibitors has been investigated in patients with $\mathrm{mCRPC}$ that harbored DDR mutations. In the PROfound trial, de Bono et al. assessed the efficacy and safety of olaparib in patients with mCRPC harboring DDR mutations who had disease progression while receiving a new hormonal agent (e.g., enzalutamide or abiraterone). Olaparib was associated with longer PFS (median, 7.4 months vs. 3.6 months; HR, 0.34; 95\% CI, 0.25 to $0.47 ; P<0.001$ ) than either enzalutamide or abiraterone [13]. In another phase II study, Abida et al. evaluated the effect of rucaparib for the treatment of men with mCRPC associated with a deleterious alteration in BRCA or other DDR gene who have progressed after next-generation AR-directed therapy and a taxanebased chemotherapy. Rucaparib was demonstrated to be associated with good antitumor activity in patients with $\mathrm{mCRPC}$ and a deleterious BRCA alteration [14]. Although the phase 3 TRITON3 trial (ClinicalTrials.gov number, NCT02975934), which evaluates rucaparib 600 mg BID vs physician's choice of abiraterone, enzalutamide, or docetaxel in patients with $\mathrm{mCRPC}$ and a deleterious germline or somatic BRCA1, BRCA2, or ATM mutation, is going on. We predict that rucaparib will also benefit men with mCRPC harboring BRCA1 or BRCA2 alterations than abiraterone, enzalutamide, or docetaxel. These studies provide evidences that PARP inhibitors might be another promising option for patients with mCRPC that harbored DDR mutations. However, in the CARD trial, no detailed data of patients with $\mathrm{mCRPC}$ that harbored DDR mutations were reported. As cabazitaxel and PARP inhibitors all showed superior efficacy than subsequent ASTIs, it is interesting to compare the efficacy and cost-effectiveness of these two options in men with mCRPC that harbored DDR mutations in the future.

The limitations of this study should be addressed. First, the analysis was performed based on data from the CARD trial, which might not fully reflect the status in the real-world. Thus, a cost-effectiveness analysis based on real-world data should be performed when the data are available. Second, the analysis included AEs that were relatively frequent ( $>5 \%$ ), expensive to treat or substantively affected quality of life. The costs of grade 1-2 AEs and AEs with low frequency were not included in the study. Fortunately, the results of the one-way sensitivity analyses demonstrated that the economic results were not sensitive to AE-related parameters. Third, the utility scores in the analysis were derived from previously published literature, which may also undermine the robustness of our results.

\section{Conclusions}

In summary, we established a Markov model to evaluate the cost-effectiveness of cabazitaxel versus ASTIs in patients with $\mathrm{mCRPC}$ previously treated with docetaxel who had progression within 12 months while receiving ASTIs from the US payer's perspective. Based on the results of the study, cabazitaxel is unlikely to be a cost-effective treatment option compared with ASTIs in patients with mCRPC previously treated with docetaxel who had progression within 12 months while receiving the ASTIs.

\section{Abbreviations \\ ADT: Androgen deprivation therapy; LHRH: Luteinizing hormone-releasing hormone; mCRPC: Metastatic castration-resistant prostate cancer; \\ ASTIs: Androgen-signaling-targeted-inhibitors; PFS: Progression-free survival; OS: Overall survival; PD: Progressive disease; AEs: Adverse events; \\ QALYs: Quality-adjusted life-years; ICER: Incremental cost-effectiveness ratio; WTP: Willingness-to-pay; BSA: Body surface area; BSC: Best supportive care}

Acknowledgements

Not applicable.

Authors' contributions

PFZ and QL were responsible for the study conception, methodology, data analysis, draft writing and editing. DX was responsible for the design, data collection and analysis, draft writing and editing. All authors have read and approved the final manuscript. 


\section{Funding}

This work was funded by the National Natural and Scientific Foundation of China (NO. 81572988) and Science \& Technology Department of Sichuan Province Funding Project (NO.2016FZ0108, 2018SZ0117). The funding was used for the design of the study, data collection, analysis, manuscript writing and editing.

\section{Availability of data and materials}

The data generated during this study are available from the corresponding author on reasonable request.

\section{Ethics approval and consent to participate}

Not applicable.

\section{Consent for publication}

Not applicable.

\section{Competing interests}

The authors declare that they have no conflict of interests.

\section{Author details}

'Department of Medical Oncology, Cancer Center, West China Hospital, Sichuan University, Chengdu, China. ${ }^{2}$ West China Biomedical Big Data Center, West China Hospital/West China School of Medicine, Sichuan University, Chengdu, China. ${ }^{3}$ Prenatal Diagnosis Center, Department of Obstetrics and Gynecology, West China Second University Hospital, Sichuan University, Chengdu, China. ${ }^{4}$ Key Laboratory of Birth Defects and Related Diseases of Women and Children (Sichuan University), Ministry of Education, Chengdu, China.

Received: 5 July 2020 Accepted: 21 December 2020

Published online: 07 January 2021

\section{References}

1. Bray F, Ferlay J, Soerjomataram I, Siegel RL, Torre LA, Jemal A. Global cancer statistics 2018: GLOBOCAN estimates of incidence and mortality worldwide for 36 cancers in 185 countries. CA Cancer J Clin. 2018;68(6):394-424.

2. Sharifi N, Gulley JL, Dahut WL. An update on androgen deprivation therapy for prostate cancer. Endocr Relat Cancer. 2010;17(4):R305-R15.

3. Maluf FC, Smaletz O, Herchenhorn D. Castration-resistant prostate cancer: systemic therapy in 2012. Clinics. 2012;67(4):389-94.

4. Tannock IF, De Wit R, Berry WR, Horti J, Pluzanska A, Chi KN, et al. Docetaxel plus prednisone or mitoxantrone plus prednisone for advanced prostate cancer. N Engl J Med. 2004:351(15):1502-12.

5. De Bono JS, Oudard S, Ozguroglu M, Hansen S, Machiels J-P, Kocak I, et al. Prednisone plus cabazitaxel or mitoxantrone for metastatic castrationresistant prostate cancer progressing after docetaxel treatment: a randomised open-label trial. Lancet. 2010;376(9747):1147-54.

6. Kantoff PW, Higano CS, Shore ND, Berger ER, Small EJ, Penson DF, et al. Sipuleucel-T immunotherapy for castration-resistant prostate cancer. N Engl J Med. 2010;363(5):411-22

7. De Bono JS, Logothetis CJ, Molina A, Fizazi K, North S, Chu L, et al. Abiraterone and increased survival in metastatic prostate cancer. N Engl J Med. 2011;364(21):1995-2005.

8. Scher HI, Fizazi K, Saad F, Taplin M-E, Sternberg CN, Miller K, et al. Increased survival with enzalutamide in prostate cancer after chemotherapy. N Engl J Med. 2012;367(13):1187-97.

9. Sweeney CJ, Chen Y-H, Carducci M, Liu G, Jarrard DF, Eisenberger M, et al. Chemohormonal therapy in metastatic hormone-sensitive prostate cancer. N Engl J Med. 2015;373(8):737-46.

10. James ND, Sydes MR, Clarke NW, Mason MD, Dearnaley DP, Spears MR, et al. Addition of docetaxel, zoledronic acid, or both to first-line long-term hormone therapy in prostate cancer (STAMPEDE): survival results from an adaptive, multiarm, multistage, platform randomised controlled trial. Lancet. 2016;387(10024):1163-77.

11. Fizazi K, Tran N, Fein L, Matsubara N, Rodriguez-Antolin A, Alekseev BY, et al. Abiraterone plus prednisone in metastatic, castration-sensitive prostate cancer. N Engl J Med. 2017;377(4):352-60.

12. Davis ID, Martin AJ, Stockler MR, Begbie S, Chi KN, Chowdhury S et al. Enzalutamide with standard first-line therapy in metastatic prostate cancer. N Engl J Med. 2019;381(2):121-31.
13. de Bono J, Mateo J, Fizazi K, Saad F, Shore N, Sandhu S, et al. Olaparib for metastatic castration-resistant prostate Cancer. N Engl J Med. 2020;382(22): 2091-102.

14. Abida W, Patnaik A, Campbell D, Shapiro J, Bryce AH, McDermott R et al. Rucaparib in Men With Metastatic Castration-Resistant Prostate Cancer Harboring a BRCA1 or BRCA2 Gene Alteration. J Clin Oncol. 2020;38(32): 3763-72.

15. Loriot Y, Bianchini D, lleana E, Sandhu S, Patrikidou A, Pezaro C, et al. Antitumour activity of abiraterone acetate against metastatic castrationresistant prostate cancer progressing after docetaxel and enzalutamide (MDV3100). Ann Oncol. 2013;24(7):1807-12

16. Attard G, Borre M, Gurney H, Loriot Y, Andresen-Daniil C, Kalleda R, et al. Abiraterone alone or in combination with enzalutamide in metastatic castration-resistant prostate cancer with rising prostate-specific antigen during enzalutamide treatment. J Clin Oncol. 2018;36(25):2639.

17. Maines F, Caffo O, Veccia A, Trentin C, Tortora G, Galligioni E, et al. Sequencing new agents after docetaxel in patients with metastatic castration-resistant prostate cancer. Crit Rev Oncol Hematol. 2015;96(3):498-506.

18. Mezynski J, Pezaro C, Bianchini D, Zivi A, Sandhu S, Thompson E, et al. Antitumour activity of docetaxel following treatment with the CYP17A1 inhibitor abiraterone: clinical evidence for cross-resistance? Ann Oncol. 2012;23(11):2943-7.

19. de Wit R, de Bono J, Sternberg CN, Fizazi K, Tombal B, Wülfing C et al. Cabazitaxel versus abiraterone or enzalutamide in metastatic prostate cancer. N Engl J Med. 2019;381(26):2506-18.

20. Dieleman JL, Templin T, Sadat N, Reidy P, Chapin A, Foreman K, et al. National spending on health by source for 184 countries between 2013 and 2040. Lancet. 2016;387(10037):2521-35.

21. Dieleman JL, Campbell M, Chapin A, Eldrenkamp E, Fan VY, Haakenstad A, et al. Future and potential spending on health 2015-40: development assistance for health, and government, prepaid private, and out-of-pocket health spending in 184 countries. Lancet. 2017;389(10083):2005-30.

22. Barqawi YK, Borrego ME, Roberts MH, Abraham I. Cost-effectiveness model of abiraterone plus prednisone, cabazitaxel plus prednisone and enzalutamide for visceral metastatic castration resistant prostate cancer therapy after docetaxel therapy resistance. J Med Econ. 2019;22(11):1202-9.

23. Criss SD, Mooradian MJ, Watson TR, Gainor JF, Reynolds KL, Kong CY. Costeffectiveness of Atezolizumab Combination Therapy for First-Line Treatment of Metastatic Nonsquamous Non-Small Cell Lung Cancer in the United States. JAMA Netw Open. 2019;2(9):e1911952-e.

24. Zhong L, Pon V, Srinivas S, Nguyen N, Frear M, Kwon S, et al. Therapeutic options in docetaxel-refractory metastatic castration-resistant prostate cancer: a cost-effectiveness analysis. PLoS One. 2013;8(5):e64275-e.

25. Nguyen C, Lairson DR, Swartz MD, Du XL. Cost-effectiveness of adding androgen deprivation therapy to radiation therapy for men with advanced prostate Cancer from a U.S. Payer's perspective. J Manag Care Spec Pharm. 2019:25(2):225-34

26. Diaby $\mathrm{V}$, Adunlin G, Montero AJ. Survival modeling for the estimation of transition probabilities in model-based economic evaluations in the absence of individual patient data: a tutorial. PharmacoEconomics. 2014;32(2):101-8.

27. Chen Q, Ayer T, Nastoupil LJ, Rose AC, Flowers CR. Comparing the costeffectiveness of rituximab maintenance and radioimmunotherapy consolidation versus observation following first-line therapy in patients with follicular lymphoma. Value Health. 2015;18(2):189-97.

28. Gong CL, Hay JW. Cost-effectiveness analysis of abiraterone and sipuleucel-T in asymptomatic metastatic castration-resistant prostate cancer. J Natl Compr Cancer Netw. 2014;12(10):1417-25

29. Wu B, Zhang Q, Sun J. Cost-effectiveness of nivolumab plus ipilimumab as first-line therapy in advanced renal-cell carcinoma. J Immunother Cancer. 2018;6(1):124

30. Ramamurthy C, Handorf EA, Correa AF, Beck JR, Geynisman DM, editors. Cost-effectiveness of abiraterone versus docetaxel in the treatment of metastatic hormone naïve prostate cancer. Urol Oncol. 2019;37(10):688-95.

31. Dan R, Allen EMV, Wu YM, Schultz N, Lonigro RJ, Mosquera JM, et al. Integrative clinical genomics of advanced prostate Cancer. Cell. 2015;161(5):1215-28.

32. Pritchard CC, Mateo J, Walsh MF, De Sarkar N, Abida W, Beltran H, et al. Inherited DNARepair Gene Mutations in Men with Metastatic Prostate Cancer. N Engl J Med. 2016;375(5):443-53.

\section{Publisher's Note}

Springer Nature remains neutral with regard to jurisdictional claims in published maps and institutional affiliations. 